\title{
NOTE
}

\section{$g$-Factors of the SH $(0-0)$ Band and SH Upper Limit in Comet P/Brorsen-Metcalf (1989o)}

\author{
Sang J. Kim and Michael F. A'Hearn \\ Astronomy Department. University of Maryland. College Park, Maryland 20742
}

Received October 8. 1991; revised March 20, 1992

Since $\mathrm{H}_{2} \mathrm{~S}$ was detected in Comets Austin (1989c1) and Levy (1990c) in the microwave range, there has been increasing interest in searching for $\mathrm{SH}$, which is the prime dissociative product of $\mathrm{H}_{2} \mathrm{~S}$. We present $\mathrm{g}$-factors for the $A-X(0-0)$ band of $\mathrm{SH}$ as a function of heliocentric velocity at $r=1.0 \mathrm{AU}$. We derive an upper limit production rate, $Q(\mathrm{SH}) / Q\left(\mathrm{H}_{2} \mathrm{O}\right)<0.017$, for Comet Brorsen-Metcalf (1989o) and calculate a dissociative lifetime of $105 \mathrm{sec}$ at a heliocentric distance, $r=1.0 \mathrm{AU}$, and at a heliocentric velocity, $\nu(r)=-28.5 \mathrm{~km} \mathrm{sec}^{-1}$. 1992 Academic Press. Inc.

Introduction. Colom ef al. (1990) recently reported the first detection of $\mathrm{H}_{2} \mathrm{~S}$ in the millimeter-wavelength range of Comet Austin $(1989 \mathrm{c} 1)$. Subsequently Crovisier at al. (1991) presented production rates of $\mathrm{H}_{2} \mathrm{~S}$ for Comets Austin and Levy (1990) derived from their microwave data. Marconi et al. (1990) first identified $\mathrm{H}_{3} \mathrm{~S}^{-}$in a mass spectrum of Comet Halley obtained with the heavy ion analyzer (PICCA) on board the Giotto spacecraft and attributed its existence to protonation of $\mathrm{H}_{2} \mathrm{~S}$. It is. therefore, of interest to search for $\mathrm{SH}$, which is the prime dissociative product of $\mathrm{H}_{2} \mathrm{~S}$. Krishna Swamy and Wallis (1986) and Wallis and Krishna Swamy (1987) tentatively identified the $B-X$ bands of $S H$ in the 1670-1920 $A$ range in the IUE spectra of faint comets and of Comet Halley, respectively. The suspected features in those spectra are, however, hampered by the typical IUE noise (e.g., Kim and A Hearn 1991). The recent availability of good. high-resolution, groundbased spectra covering the spectral range including the SH $A-X$ transitions ( $O^{\circ}$ Dell $e t$ al. 1990) provides additional stimulus for estimating the expected strength of the emission by SH.

g-factors of the $S H A-X(0-0)$ band. The individual lines of the $\mathrm{SH}$ $A-X(0-0)$ band have been observed in ahsorption, and their line positions were presented by Ramsay (1952). We used his line positions in our $g$-factor calculations because the line positions are sufficiently accurate for calculation of the Swings effect, and they are the best available in the literature.

The $A-X$ system is the same type of electronic transition as is the $\mathrm{OH}$ $A-X$ system. For the Hönl-London (H-L) factors of this band, we adopted an updated list of the H-L factors for the ' - - - 'II system of the $\mathrm{OH} A-X$ band presented in Appendix B of Schleicher and A'Hearn (1982)

Friedl of al. (1983) found experimentally that predissociation of $\mathrm{SH}$ occurs throughout the rotational levels of the $0-0$ band of the $A-X$ system, although previously only the $v^{\prime} \geq 1$ levels of the $A$ state had been thought to predissociate significantly. They found that the predissociative and radiative lifetimes are 3 and $820 \mathrm{nsec}$. respectively. Senekowitsch at al. (1985) subsequently revised the radiative lifetime to be $704 \mathrm{nsec}$. Since most of the radicals predissociate subsequent to their first excitation to the $A$ state, fluorescent equilibrium will not he established and the emission spectrum will be controlled by the Swings effect operating on a ground-state population. The general formula for the $g$-factor $\left(s^{1}\right)$ for a transition undergoing predissociation can be expressed by

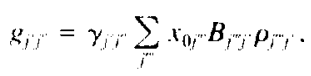

where the summation is restricted by the selection rule for the rotational transitions. $x_{0 \prime}$ is the fractional population of the $j^{\prime \prime \prime}$ rotational level in the ground state, $\gamma_{i, i}$ is the branching ratio for radiative decay from the $j$ ' rotational level of the excited state to the $j^{\prime \prime}$ rotational level of the ground state, $B_{i, j}$ is the Einstein $B$ coefficient, and $\rho_{i, j}$, is the solar radiation density. There is major uncertainty as to what should be used for the populations of the ground-state rotational levels, $x_{0, \cdots}$. The following is a discussion of the possible population of the ground state and corresponding temperature of $\mathrm{SH}$.

Hawkins and Houston (1980) conducted laboratory experiments on the photodissociation of $\mathrm{H}_{2} \mathrm{~S}$ and measured the rotational temperatures of SH. They found that the SH radicals are formed a little warmer (about $40 \mathrm{~K}$ greater) than their parents when their parents are at room temperature. Hawkins and Houston (1982) performed the same experiment for $\mathrm{H}_{2} \mathrm{~S}$ at $3 \mathrm{~K}$ and found that the rotational temperature of the nascent $\mathrm{SH}$ is about $150 \mathrm{~K}$. This indicates that if the parents are very cold, the nascent SH becomes much warmer $(150 \mathrm{~K})$ than the parents. From the results of Hawkins and Houston. the rotational temperature of the nascent SH seems to be greater than $150 \mathrm{~K}$ regardless of the $\mathrm{H}_{2} \mathrm{~S}$ temperature.

Crovisier et al. (1991) measured a rotational temperature of $40 \mathrm{~K}$ for $\mathrm{H}_{2} \mathrm{~S}$ in Comet Austin at about $1 \mathrm{AU}$ heliocentric distance. Although the rotational temperature was not measured, we expect a low rotational temperature for this molecule in Comet $\mathrm{B}-\mathrm{M}$ because rotationally excited $\mathrm{H}_{2} \mathrm{~S}$ relaxes rapidly (D. Bockelee-Morvan, private communication, 19911. From these arguments. we can say that the temperature of the nascent SH is greater than $150 \mathrm{~K}$ and is likely to be less than $400 \mathrm{~K}$ because of the probably low excitation temperature of $\mathrm{H}_{2} \mathrm{~S}$.

In order to estimate the changes in the rotational populations of $\mathrm{SH}$ expected after formation but prior to fluorescence, we roughly calculated rotational transition probabilities of $\mathrm{SH}$ as follows. The $\mathrm{OH}$ and $\mathrm{SH}$ 
ground states have the same transition types, and therefore the only major differences are the frequency and dipole moment. The radiative transition probability is proportional to the square of the dipole moment and the cube of the frequency. The dipole moments of $\mathrm{SH}$ and $\mathrm{OH}$ are 0.7580 and $1.6676 \mathrm{D}$, respectively, and the rotational $B$ constants are 9.46 and $18.5 \mathrm{~cm}^{-1}$, respectively. Burdyuzha and Varshalovich (1973) presented Einstein $A$ coefficients for the hyperfine transitions of $\mathrm{OH}$. We scale down these values using the ratios of the dipole moments and the frequencies of $\mathrm{SH}$ and $\mathrm{OH}$. The resultant radiative lifetimes for the lowest lines in the $R$ branch are about $200 \mathrm{sec}$, which are greater than the $\mathrm{SH}$ lifetime of $\sim 100 \mathrm{sec}$ as discussed in the next section. The radiative lifetime decreases as rotational quantum number $J$ increases, and it approaches $1 \mathrm{sec}$ at around $J=25$. The radiative lifetimes for lines in other satellite branches are much greater than the SH lifetime. We conclude that the lower rotational levels of the $\mathrm{SH}$ radicals do not undergo significant rotational deexcitation (or cooling) before they are destroyed by photodissociation.

Since the $\mathrm{SH}$ is formed where densities in the coma are moderately high, collisions can also affect the distribution of populations. The recent model of Crifo (1991) predicts kinetic temperatures near $200 \mathrm{~K}$ at a distance of $4000 \mathrm{~km}$ from the nucleus, the mean distance at which $\mathrm{H}_{2} \mathrm{~S}$ photodissociates to produce the SH. Thus collisions, which would tend to produce rotational distributions in equilibrium with the kinetic temperature, will tend to produce a rotational excitation characterized by a temperature near $200 \mathrm{~K}$. We therefore will assume that the rotational distribution in the ground state can be characterized by temperatures between 150 and $400 \mathrm{~K}$. This range leads to about a $15 \%$ variation in the $g$-factor for the entire 0- 0 band and about a $50 \%$ variation for the $R_{1}+$ ${ }^{R} Q_{21}$ branch lines clustered near $3238 \AA$. These variations are still smaller than the variation caused by the Swings effect. The variation for individual lines within the band, however, is greater than that caused by the Swings effect.

The other parameters needed to solve Eq. (1) are much better determined. The branching ratio, $\gamma_{i j j^{\prime \prime}}$, can be expressed in terms of the Einstein $A$ coefficients for the downward transitions coupled with the dissociative lifetime of the upper state as follows:

$$
\gamma_{j j^{\prime}}=\frac{A_{i j^{\prime \prime}}}{\left(\mathrm{I} / \tau_{\mathrm{d}}\right)+A_{j j^{\prime \prime}}+A_{j j^{\prime \prime+1}}+A_{j^{\prime \prime \prime}-1}} .
$$

Here $A_{j,}$ is the Einstein $A$ coefficient and $\tau_{\mathrm{d}}$ is the dissociative lifetime of the $v^{\prime}=0$ state in the $A$ state. The equation is written for a $Q$-branch line, and the subscripts in the denominator differ for $P$ and $R$ branches. In all cases, the branching ratio can be approximated as $A_{j j} \tau_{\mathrm{d}}$ because $1 / \tau_{\mathfrak{f}}$ dominates the term in the denominator.

$B_{I^{\prime \prime}}$ is the Einstein coefficient for radiative absorption in a given transition and can be expressed in terms of $A(0-0)$, the Einstein coefficient for spontaneous emission in the entire band, as follows:

$$
B_{j^{\prime \prime}}=\frac{A(0-0) \mathrm{H}-\mathrm{L}}{8 \pi h \omega_{0-0}^{?}\left(2 j^{\prime \prime \prime}+1\right)}
$$

where $h$ is Planck's constant. $\omega_{(1-0)}$ is the frequency of the band head in units of $\mathrm{cm}^{-1}$, and $\mathrm{H}-\mathrm{L}$ is the Hönl-London factor for the transition taken, as noted above, from Schleicher and A'Hearn (1982).

The radiation density at each transition, $\rho_{j^{\prime \prime} j^{\prime}}$, is taken directly from A'Hearn ot al. (1983) with allowance for the Swings effect.

Using the above equations, $g$-factors for Comet B-M (1989o) have been calculated for a heliocentric distance, $r=1.0 \mathrm{AU}$, and for a heliocentric velocity, $v(r)=-28.5 \mathrm{~km} \mathrm{sec}^{-1}$. A model spectrum made from these $g$-factors with a $1-\AA$ spectral resolution is presented in Fig.
1. The $R_{1}+{ }^{R} Q_{21}$ branch occurs at $3238 \AA$, consisting of about 15 individual lines. In Fig. 2 we present $g$-factors for this branch as a function of heliocentric velocity at $r=1 \mathrm{AU}$. The $g$-factor for this branch is $6.2 \times 10^{-6} \mathrm{sec}^{-1}$ for $v(r)=0 \mathrm{~km} \mathrm{sec}^{-1}$ and $7.8 \times 10^{-6} \mathrm{sec}^{-1}$ for $v(r)=-28.5 \mathrm{~km} \mathrm{sec}^{-1}$. The $g$-factor of the entire $0-0$ band ranges from $2.5 \times 10^{-5}$ to $4.1 \times 10^{-5} \mathrm{sec}^{-1}$ for the heliocentric velocity between -80 and $80 \mathrm{~km} \mathrm{sec}^{-1}$ at a constant temperature of $200 \mathrm{~K}$. The total $\mathrm{g}$ factor also varies between $3.4 \times 10^{-5}$ and $4.4 \times 10^{-5} \mathrm{sec}^{-1}$ for the temperature range of $50-400 \mathrm{~K}$ at a heliocentric velocity of $0.0 \mathrm{~km} \mathrm{sec}-1$. Our values are significantly lower than the value $\left(2.32 \times 10^{-3} \mathrm{sec}^{-1}\right)$ derived by Krishna Swamy and Wallis (1988), who apparently did not consider predissociation in the $A-X$ band.

Krishna Swamy and Wallis (1986) and Wallis and Krishna Swamy (1987) tentatively identified the $B-X$ band of $\mathrm{SH}$ in the $1670-1920 \AA$ range in the IUE spectra of certain comets. Krishna Swamy and Wallis (1988) derived the $g$-factors of the $B-X$ bands to be less than $5 \times 10^{-8}$ $\mathrm{sec}^{-1}$, which is so low that the presence of the band in the noisy IUE spectra is doubtful. They also did not consider possible predissociation in the $B-X$ band. According to the absorption spectrum of the $B-X$ band presented in Fig. 1 of Morrow (1966), the absorption linewidths $(\sim 0.8$ $\left.\mathrm{cm}^{-1}\right)$ are wider than the uncertainty $\left( \pm 0.1 \mathrm{~cm}^{-1}\right)$ in the line positions. This suggests that predissociation occurs through these lines, and the predissociation will lower the derived $g$-factors even more, rendering the tentative detection even more doubtful.

Upper limits of $S H$ in Comet P/Brorsen-Metcalf and lifetimes of $S H$. In order to calculate the upper limits of $\mathrm{SH}$ in comets. the lifetimes of $\mathrm{H}_{2} \mathrm{~S}$ and $\mathrm{SH}$ are required. Crovisier et al. (1991) estimated an $\mathrm{H}_{2} \mathrm{~S}$ lifetime of $4000 \mathrm{sec}$ at $1 \mathrm{AU}$ using an absorption spectrum of Lee et al. (1987).

The dissociation rate of $\mathrm{SH}$ through the $0-0$ transitions can be easily derived from Eq. (1) and is given by

$$
D_{\mathrm{r}}(0-())=\sum_{j^{m+m}} x_{0 j^{+}} B_{H^{\prime} j} \rho_{7^{\prime \prime \prime} j^{\prime}}
$$

Here we have used the lifetimes given previously to assume that the branching ratio for dissociation is nearly unity. The total dissociation rate through the $0-0,1-0$, and $2-0$ bands is the sum of $D_{r}(0-0), D_{r}(1-0)$, and $D_{r}(2-0) . D_{r}(0-0)$ at $1 \mathrm{AU}$ is $8.20 \times 10^{-3} \mathrm{sec}^{-1}$ for $v(r)=-28.5 \mathrm{~km}$ $\sec ^{-1}$ and $5.78 \times 10^{-3} \mathrm{sec}^{-1}$ for $v(r)=0 \mathrm{~km} \mathrm{sec}-1$. According to Franck-Condon (F-C) factor calculations by Nicholls et al. (1960), which, although old, is the only available source, the $F-C$ factors of the $0-0,1-0$, and $2-0$ transitions are $0.74,0.20$, and 0.04 , respectively. The $0-0,1-0$, and $2-0$ bands occur at approximately 3250,3070 , and 2923 $\AA$, where the solar radiation densities are about $1.2 \times 10^{-20}, 6.0 \times 10^{-21}$, and $5.2 \times 10^{-21} \mathrm{erg}^{-3} \mathrm{~Hz}^{-1}$, respectively. Considering the $\mathrm{F}-\mathrm{C}$ factors and the radiation densities, we obtain a total dissociation rate of $9.50 \times 10^{-3} \mathrm{sec}^{-1}$ at $1 \mathrm{AU}$ for $v(r)=-28.5 \mathrm{~km} \mathrm{sec}^{-1}$. The SH lifetime is, therefore, about $105 \mathrm{sec}$ at $1 \mathrm{AU}$ for $v(r)=-28.5 \mathrm{~km} \mathrm{sec}^{-1}$.

O'Dell ct al. (1991) presented a spectrum of Comet P/Brorsen-Metcalf covering the $3000-3600 \AA$ range with $1-\AA$ s spectral resolution. From Fig. 4 of $O^{\prime}$ Dell et al., we obtain an upper limit flux of $5 \times 10^{-17} \mathrm{erg} \mathrm{cm}^{-2}$ $\mathrm{sec}^{-1}$ pixel $^{-1}$ for the $3238-\AA$ band of SH. Since this flux is an average over the entrance slit, 1.28 by $139 \operatorname{arcsec}(1.28$ arcsec per pixel), the total flux, $F$, within the slit is $5.4 \times 10^{-15} \mathrm{erg} \mathrm{cm}^{-2} \mathrm{sec}^{-1}$. At the time of the observations, the average heliocentric distance was $1.0 \mathrm{AU}$, the average geocentric distance was $0.645 \mathrm{AU}$, and the average heliocentric velocity was $-28.5 \mathrm{~km} \mathrm{sec}^{-1}$. Using the formula

$$
N(\text { SH per slit })<\frac{4 \pi \Delta^{2} F}{g h \nu}
$$

we obtain $N$ (SH per slit) $<1.3 \times 10^{29}$ for the slit area of $600 \times 32,600$ $\mathrm{km}$. Here, $\Delta$ is the geocentric distance, $g$ is the $g$-factor, and $\nu$ is the 


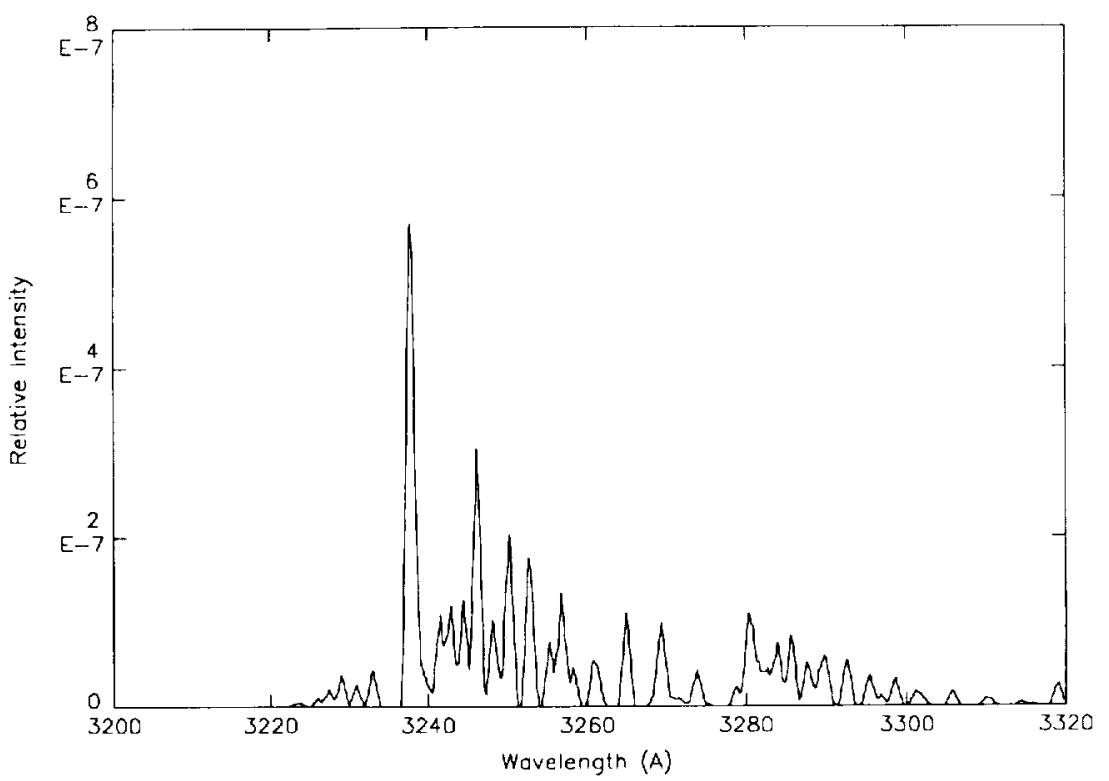

FIG. 1. A model spectrum of the $0-0$ band of the $A-X$ system with a $1-\AA$ slitwidth for a heliocentric velocity, $v(r)=-28.5 \mathrm{~km}$ sec ${ }^{-1}$. at $r=\mathrm{I} A \mathrm{AU}$.

frequency. Using the $\mathrm{H}_{2} \mathrm{~S}$ lifetime of $4000 \mathrm{sec}$ and the SH lifetime of 105 sec, and a Haser model (Haser 1957) with $v=1 \mathrm{~km} \mathrm{sec}^{-1}$ for $\mathrm{SH}$ and $\mathrm{H}_{2} \mathrm{~S}$, we obtain a production rate of $\mathrm{SH} . Q(\mathrm{SH})$, which is less than $6.7 \times 10^{27} \mathrm{sec}^{-1}$. Using the $\left.\mathrm{OH}(0)-0\right)$ observations of Comet B-M by ODell a al. we determined $Q\left(\mathrm{H}_{2} \mathrm{O}\right)$ to be about $4.0 \times 10^{29} \mathrm{sec}^{-1}$ using at Haser model with $v=1 \mathrm{~km} \mathrm{sec}^{-1}$. We derive that $Q(\mathrm{SH}) / Q\left(\mathrm{H}_{2} \mathrm{O}\right)$ is about $<0.017$.

Crovisier et al. (1991) reported a production rate of $\mathrm{H}_{2} \mathrm{~S}, Q\left(\mathrm{H}_{2} \mathrm{~S}\right)$ $Q\left(\mathrm{H}_{2} \mathrm{O}\right)=2 \times 10^{-3}$, for both Comets Austin and Levy (1990) using their microwave observations. Our upper limit for Comet B-M is thus consistent with the results of Crovisier e' al. for Comets Austin and Levy. Detection of $\mathrm{SH}$, which should require an order of magnitude

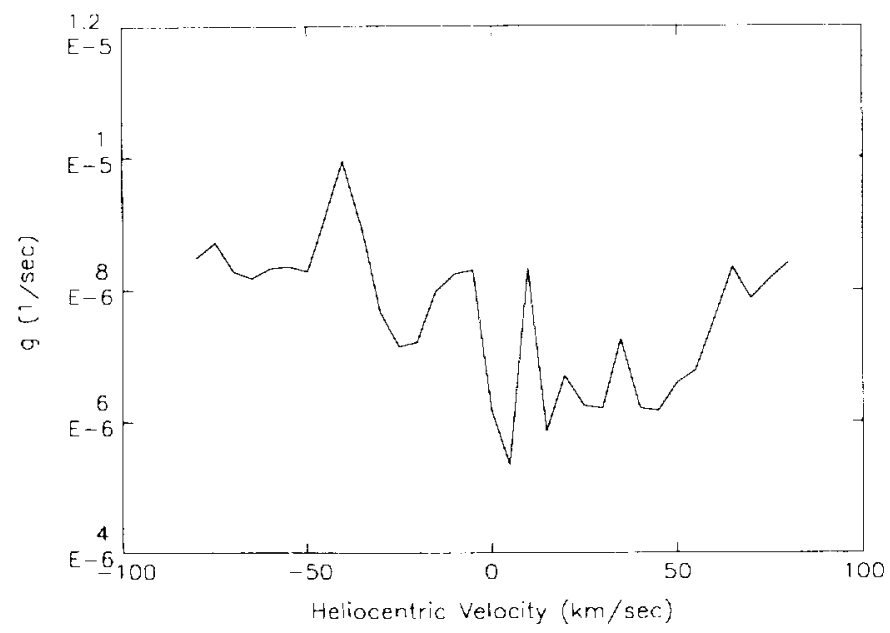

FIG. 2. $g$-Factors as a function of heliocentric velocity at $r=1 \mathrm{AU}$ for the $R_{1}+{ }^{R} Q_{21}$ branch which produces the sharp feature at $3238 \AA$. and which consists of about 15 individual lines. greater sensitivity, would be valuable in testing the models used to interpret the observations of $\mathrm{H}_{2} \mathrm{~S}$.

\section{ACKNOWLEDGMENTS}

We thank J. Crovisier for giving us encouragement to publish this paper. We also thank W. D. Cochran for his discussions on the signalto-noise ratios of the Comet B-M data. We are grateful to D. BockeleeMorvan for her detailed and critical comments on this paper as a referee. This work was supported by NASA Grant NAGW-9(12.

\section{REFERENCES}

A Hearn, M. F., J. T. Ohlmacher, and D. G. Schificher, 1983. A High Resolution Solur Atlas for Fluorescence Calculations. Tech. Rep. TR-AP83-044, Dept. of Physics and Astronomy. University of Maryland.

BURDYUzha, V. V., AND D. A. VARSHalovich 1973. Infrared and radio transition probabilities of $\mathrm{OH}$ and $\mathrm{CH}$. Sov. Astron. 16, 980-983.

Colom, P. D. Despois, D. Bockfi.e.-Morvan, J. Crovisifr, and G. PAUBERT 1990. Millimetre observations of comets P/Brorsen-Metcalf (1980o) and Austin (1989c1) with the IRAM 30-m radio telescope. Proc. Workshop: Observations of Recent Comets (1990), Albuquerque, NM. June 15-16. 1990. pp. 80-85.

CrIFo, J. F. 1991. Cometary gas phase chemistry taking into account homogeneous and ion-induced water recondensation. To be published.

Crovisier, J., D. Despois, D. Bockelef-Morvan, P. Colom, and G. Paubert 1991. Microwave observations of hydrogen sulfide and searches for other sulfur compounds in Comets Austin (1989c1) and Levy (1990c). 93, 246-258.

Frifdi. R. R., W. H. Brunf, and J. G. ANderson 1983. Radiative and predissociative lifetimes of the $V^{\prime \prime}=0$ level of the $A^{2}{ }^{+}$state of SH and SD from chemical and spectroscopic studies. J. Chem. Phys. 79, $4227-4236$. 
Haser, L. 1957. Distribution d'Intensite dans la tête d'une comête. Bull Cl. Sci. Acad. R. Belg. 43, 740-750.

HaWkins, W. G., aND P. L. Houston $1980.193 \mathrm{~nm}$ photodissociation of $\mathrm{H}_{2} \mathrm{~S}$ : The $\mathrm{SH}$ internal energy distribution. J. Chem. Phys. 73, 297-302.

Hawkins, W. G., and P. L. Houston 1982. Rotational distributions in the photodissociation of bent triatomic: $\mathrm{H}_{2} \mathrm{~S}$. J. Chem. Phys. 76, $729-731$.

KIM, S. J., and M. F. A'Hearn 1991. Upper limits of $\mathrm{SO}^{\prime}$ and $\mathrm{SO}_{2}$ in comets. Icurus 90, 79-95.

Krishna Swamy, K. S., and M. K. Wal.I.ts 1986. Sulphur compounds in comets. In New Insights in Astrophysics, ESA SP-243, pp. 133-136.

Krishna Swamy, K. S., and M. K. Wal.tis 1988. Resonance fluorescence calculations for some sulphur diatomics in comets. Astron. Astrophys. 74, 227-232.

LEE, L. C., X. WANG, AND M. Suto 1987. Quantitative photoabsorption and fluorescence spectroscopy of $\mathrm{H}_{2} \mathrm{~S}$ and $\mathrm{D}, \mathrm{S}$ at $49-240 \mathrm{~nm} . J$. Ch'm. Phys. 86, 4353-4361.

Marconi, M. L.. D. A. Mendis, A. Korth, R. P. Lin, D. L. MitchELI. AND H. REME 1990. The identification of $\mathrm{H}_{3} \mathrm{~S}^{-}$with the ion of mass per charge $(\mathrm{m} / \mathrm{q}) 35$ observed in the coma of comet Halley. Astrophys. J. 352, L17-L20.
Morrow, B. A. 1966. The absorption spectrum of SH and SD in the vacuum ultraviolet. Can. J. Phys. 44, 2447-2459.

Nicholls, R. W., P. A. Fraser, W. R. Jarmain, and R. P. McEachRAN 1960. Vibrational transition probabilities of diatomic molecules: Collected results. IV. BeO, BO, $\mathrm{CH}^{+}, \mathrm{CO}, \mathrm{NO}, \mathrm{SH}, \mathrm{O}_{2}, \mathrm{O}_{2}{ }^{+}$. Astrophys. J. 131, 399-406.

o'Dell, C. R., C. O. Miller, A. L. Cochran, W. D. Cochran, C. B. Opal, AND E. S. Barker 1990. New ultraviolet spectroscopic features found in comet Brorsen-Metcalf (19890). Astrophys. J. 368, 616-621.

RamSAY, D. A. 1952. Absorption spectra of SH and SD produced by flash photolysis of $\mathrm{H}_{2} \mathrm{~S}$ and $\mathrm{D}_{2} \mathrm{~S}$. J. Chem. Phys. 20, 1920-1927.

SChI.eicher, D. G., AND M. F. A Hearn 1982. OH fluorescence in comets: Fluorescence efficiency of the ultraviolet bands. Astrophys. J. 258, 864-877.

Senekowitsch, J., H-J. Werner, P. Rosmus, and E-A. Reinsch 1985. Ab initio calculations of radiative transition probabilities in $\mathrm{SH}$. $\mathrm{SH}^{+}$, and $\mathrm{SH}^{-}$. J. Chem. Phys, 83, 4661-4667.

Wallis, M. K., and K. S. Krishna Swamy (1987). Some diatomic molecules from comet P/Halley`s UV spectra near spacecraft flybys. Astron. Astrophys. 187, 329-332. 